\title{
Selección y entrenamiento de un panel en análisis sensorial de café Coffea arabica $L$.
}

Panel selection and training in the sensory analysis of coffee Coffea Arabica L.

\section{Nelson Gutiérrez G. ${ }^{1}$; Oscar Mauricio Barrera B. ${ }^{2}$}

1. Ph.D. Universidad Surcolombiana. Neiva, Colombia, ngutierrezg@usco.edu.co

2. Ingeniero Agrícola. Universidad Surcolombiana. Neiva, Colombia, osmabber@gmail.com

Citar: GUTIERREZ, N.; BARRERA, 0. 2015. Selección y entrenamiento de un panel en análisis sensorial de café Coffea arabica L. Rev. Cienc. Agr. 32(2):77-87.

Fecha de recepción: Febrero 7 de 2015

Fecha de aceptación: Agosto 03 de 2015

\section{RESUMEN}

El propósito de éste trabajo, fue definir una metodología para el entrenamiento de un panel para la evaluación sensorial de cafés especiales, ajustado a la metodología de la Asociación Americana de Cafés Especiales (SCAA). El proceso de entrenamiento incluyó la selección, motivación, fundamentación teórica y la aplicación de pruebas básicas de aromas y sabores y pruebas específicas de evaluación descriptiva de muestras de café; el tiempo total de entrenamiento fue de tres meses. El panel estuvo conformado por diez jueces que evaluaron 21 muestras de café en tres sesiones diferentes de cuatro horas cada una, se utilizaron pruebas de " $t$ " de Student de comparación de muestras independientes y análisis clúster para comprobar la efectividad de la metodología propuesta para el entrenamiento. Los resultados mostraron que después de la tercera sesión de adiestramiento correspondientes a 12 horas en la etapa de entrenamiento específico, no se encontraron diferencias estadísticamente significativas $(\mathrm{P}>0,05)$ entre los juicios emitidos por el panel en proceso de entrenamiento y las valoraciones de los jueces expertos; los diagramas de dispersión resultantes del análisis clúster permitieron comprobar la efectividad de la metodología propuesta.

Palabras clave: Análisis sensorial, entrenamiento de un panel sensorial, calidad sensorial de café. 


\begin{abstract}
The purpose of this paper was to define a panel training methodology in the sensory evaluation of specialty coffees using the Specialty Coffee Association of America (SCAA) methodology. The training process included selection, motivation, theoretical support, basic tests in flavor and aroma, and specific tests in the descriptive analysis of coffee samples. The training time was three months. Twenty one coffee samples were evaluated in three sessions of four hours each; " $t$ " Student tests and cluster analysis were carried out to evaluate the effectiveness of the training methodology. The results showed that after the third training session, corresponding to 12 hours in the stage of specific training, no statistically significant differences $(p>0,05)$ were found between the judgments given by expert panel and the panel in training process; the dispersion diagrams from the cluster analysis proved the effectiveness of the training methodology.
\end{abstract}

Key words: Sensory analysis, sensory panel training, coffee sensory quality.

\section{INTRODUCCIÓN}

El café se produce en 77 países, la bebida es muy apreciada por su aroma, sabor y por su efecto estimulante (Puerta, 2009). Las cualidades organolépticas del café han sido reconocidos por más de cincuenta años (Henao, 2009). Las propiedades organolépticas del café se originan en la presencia de no menos de 850 compuestos, que han sido identificados tanto en café verde como en café tostado. Los compuestos volátiles son en su mayoría los responsables del aroma y sabor del café (Bohórquez et al, 2005). Éstos pueden determinar la calidad final en taza y definir diferencias según sea su origen. Los grados de calidad o normas de calidad se han desarrollado para ayudar a la comercialización de alimentos frescos y procesados. La ruta usual para el desarrollo de estas normas se inició con la definición de los parámetros de calidad que se pueden medir; las mediciones deben ser realizadas por el mejor método disponible, científicamente validado, y totalmente correlacionado con la manera como los consumidores apreciarán tal nivel de calidad del producto. El desarrollo de los sistemas de clasificación sensorial no es una tarea fácil y debe hacerse de forma individual para cada producto; del mismo modo, la calidad sensorial debe ser evaluada por paneles que han sido entrenados adecuadamente para identificar plenamente, definir y comprender las características sensoriales que determinan dicha calidad (Feria, 2002).

El café es uno de los pocos productos agroalimentarios que es valorado con base en descriptores cualitativos, donde a mejor calidad, mayor será el precio que se obtenga. Esta calidad, sin embargo, depende de varios factores que se relacionan en todas las etapas de la producción de café, desde la selección de la variedad hasta la elaboración de la bebida (Magalhães, 2002). Es gracias a sus características y atributos que se convirtió en la bebida más popular en el mundo después del agua, pero presenta diferencias entre los países consumidores (Rendón et al., 2007).

La evaluación de la calidad del café se hace mediante la prueba de taza, en la que tradicionalmente son evaluadas de 3-10 tazas de café de una misma muestra, preparada de la misma manera; un panel de evaluadores entrenados son responsables de la prueba y en cada sesión pueden ser evaluadas más de 200 tazas por día. Estos catadores desarrollan una capacidad única para reconocer los defectos y la calidad en las bebidas de café, debido a la experiencia adquirida durante 
varios años. Rendón et al. (2007) mencionan que atributos como el aroma, sabor, acidez y el cuerpo son las características sensoriales que describen la calidad del café, siendo un café de calidad superior con una intensidad equilibrada de acidez y aroma, buen sabor y cuerpo medio.

Los métodos sensoriales descriptivos identifican y cuantifican las intensidades de los atributos evaluados, para valorar la intensidad de los atributos los jueces deben recibir un entrenamiento intensivo que les permite cuantificar de manera precisa los atributos (Rendón et al., 2007). El uso de individuos entrenados (paneles), es equivalente al uso de cualquier instrumento científico para la medición de las características que están asociadas con la calidad de un producto alimenticio (Feria, 2002). En Colombia se han desarrollado las normas GTC 245 y GTC 246 como una guía general para la selección, entrenamiento y seguimiento de evaluadores, pero las normas mencionadas se ajustan a la evaluación sensorial para la aceptación y el mercadeo de productos, mientras que la metodología SCAA se utiliza para perfilar y calificar cuantitativamente muestras de cafés especiales. Diferentes estudios se han desarrollado para definir las metodologías de entrenamiento en paneles sensoriales y definir la forma de evaluar la eficacia del entrenamiento, como el caso de Callejo et al. (2015) quienes mediante pruebas triangulares realizaron entrenamiento de un panel sensorial para evaluar productos de panificación, Kreutzmann et al. (2007) realizaron un entrenamiento a un panel sensorial para evaluar diferentes atributos sensoriales en muestras de zanahoria y Montenegro et al. (2008) definieron la metodología para entrenar un panel para la evaluación sensorial de mieles chilenas. Para el caso específico de entrenamiento de un panel para la definición del perfil sensorial de muestras de cafés especiales, solamente se reporta el estudio realizado por Henao (2009).

El objetivo de este trabajo fue definir una metodología para entrenar un panel sensorial que pueda evaluar los diferentes atributos de calidad definidos por la Asociación Americana de Cafés Especiales para realizar pruebas de taza en cafés especiales de diferentes orígenes.

\section{MATERIALES Y MÉTODOS}

La conformación del panel y las características de los panelistas, fueron definidas de acuerdo con lo establecido por Puerta (2009), teniendo en cuenta al momento de la selección de incluir personas con interés, motivación, buena salud y disponibilidad de tiempo. En la Tabla 1 se presenta la composición del panel definido para el proceso de entrenamiento.

Para determinar la actitud de los panelistas en el reconocimiento e individualización de olores, se realizó una prueba de reconocimiento de los olores básicos que comúnmente se presentan en alimentos citados por Castro et al. (2013), en concordancia con la norma colombiana NTC 4503 (ICONTEC, 1998). Los códigos y la cantidad de material utilizado en esta prueba están presentados en la Tabla 2.

Tabla 1. Composición del panel sensorial definido para el entrenamiento.

\begin{tabular}{|c|c|c|c|}
\hline Género & Número de participantes & Edad & Nivel de educación \\
\hline Femenino & 5 & 21 - 26 años & Universitaria \\
\hline Masculino & 5 & 21 - 27 años & Universitaria \\
\hline Total & 10 & & \\
\hline
\end{tabular}


Tabla 2. Material utilizado para el test de reconocimiento de olores básicos.

\begin{tabular}{clcclc}
\hline Código & Material & $\begin{array}{c}\text { Cantidad } \\
\text { (g) }\end{array}$ & Código & Material & $\begin{array}{c}\text { Cantidad } \\
\text { (g) }\end{array}$ \\
\hline 207 & Pimienta & 5 & 568 & Cebolla & 5 \\
837 & Clavos & 5 & 257 & Ajo & 5 \\
931 & Vinagre & 5 & 381 & Papa & 5 \\
425 & Canela & 5 & 573 & Miel & 5 \\
129 & Vainilla - caramelo & 5 & 243 & Limón & 5 \\
362 & Café & 5 & 674 & Alcohol & 5 \\
643 & Nuez moscada & 5 & 165 & Cocoa & 5 \\
619 & Panela & 5 & 852 & Jabón & 5 \\
479 & Apio & 5 & & & \\
\hline
\end{tabular}

Posteriormente, se realizaron siete sesiones de entrenamiento para reconocimiento de los olores del café, para lo cual se utilizó el Kit de la nariz del café (Le Nez du Café) que consta de 36 aromas típicos en café; entre cada sesión se utilizaron muestras de café fresco molido para evitar la fatiga olfativa en los catadores (Dorri et al., 2007). Finalizadas las sesiones de entrenamiento, se aplicó un test de comprobación mediante una prueba de comparación pareada utilizando dos Kits para conformar 18 parejas, buscando encontrar aciertos y diferencias entre los pares de muestras evaluadas.
Para la identificación de sabores, se realizaron dos tipos de test: el test de umbral de detección, que consistió en determinar el umbral mínimo de percepción de diferentes sustancias diluidas en agua de manera gradual, desde concentraciones de $10^{-1}$ hasta $10^{-10}$. El segundo test aplicado correspondió al test de identificación de sabores básicos en alimentos correspondientes a dulce, salado, amargo, ácido y neutro. En las Tablas 3 y 4 se muestran las soluciones utilizadas para el test de umbral de detección y test de identificación respectivamente.

Tabla 3. Soluciones para la identificación del umbral de detección de los sabores básicos.

\begin{tabular}{llc}
\hline \multicolumn{1}{c}{ Sabor } & \multicolumn{1}{c}{ Sustancia } & Concentración $(\mathrm{g} / \mathbf{1 0 0} \mathbf{m l})^{*}$ \\
\hline Dulce & Sacarosa (azúcar comercial) & 0,8 \\
Salado & Cloruro de sodio (sal comercial) & 0,5 \\
Amargo & Cafeína Anhidra (comercial) & 0,002 \\
Acido & Ácido cítrico & 0,20 \\
Neutro & Agua destilada & - \\
\hline
\end{tabular}

*concentraciones determinadas a partir de las utilizadas para un litro (Rodríguez et al., 1995). Dulce 8 g/L; Salado 5 g/L; Amargo 0,020 g/L y Ácido 2g/L 
Tabla 4. Cantidad de soluto utilizada para la identificación básica de sabores.

\begin{tabular}{llc}
\hline \multicolumn{1}{c}{ Sabor } & \multicolumn{1}{c}{ Sustancia } & Concentración g/L \\
\hline Dulce & Sacarosa (azúcar comercial) & 8 \\
Salado & Cloruro de sodio (sal comercial) & 5 \\
Amargo & Cafeína Anhidra (comercial) & 0,02 \\
Acido & Ácido cítrico & 2 \\
Neutro & Agua destilada & - \\
\hline
\end{tabular}

Terminada la fase de entrenamiento básico en aromas y sabores, se procedió al entrenamiento específico en análisis sensorial de café según la metodología de la Asociación Americana de Cafés Especiales (por sus siglas en ingles SCAA). En primer lugar, se brindó una capacitación básica para el diligenciamiento de los formatos de la SCAA y en el procedimiento de cata propiamente dicho que comprende además de una inspección visual a las muestras para observar el color del grano tostado y molido, se siguió el siguiente protocolo: después de 15 minutos de que las muestras fueron molidas se determinó la fragancia en seco, seguidamente se adicionó agua a $93^{\circ} \mathrm{C}$ en una proporción 8,25 g de café por $150 \mathrm{ml}$ de agua; la fase sólida a manera de nata sobrenadante se dejó por un periodo de cuatro minutos y se procedió a romper taza con cuchara de catación por agitación en tres tiempos, después de la agitación se permite que la espuma corra por la parte de atrás de la cuchara suavemente mientras olfatea; posteriormente cuando la muestra se enfrió hasta $71^{\circ} \mathrm{C}$, en aproximadamente 8-10 minutos de infusión, se realizó la evaluación de la bebida aspirando el líquido dentro de la boca de tal manera que cubra especialmente la lengua y paladar duro. Se realizaron tres sesiones de catación de cuatro horas cada una, con muestras de café de diferentes orígenes para cada sesión. Se dejaron intervalos de tiempo de tres días entre cada sesión. Las puntuaciones expresadas para cada atributo evaluado por el panel en entrenamiento fueron comparados con los resultados expresados por tres jueces entrenados después de consenso. Los atributos de calidad evaluados fueron fragancia/ aroma, sabor, sabor residual, acidez, cuerpo, uniformidad, dulzor, limpidez de la taza, balance e impresión global; los resultados se presentan en la escala de 6 a 10 correspondiente a la valoración de cafés especiales.

Análisis Estadístico. Para determinar el nivel de avance de los panelistas en el reconocimiento de aromas básicos, se construyó un gráfico descriptivo del porcentaje de aciertos obtenidos por el panel en función de cada sesión de entrenamiento. Para las pruebas de comparación pareadas de aroma con la nariz del café, se encontró el nivel de ajuste de las respuestas a la distribución Binomial, tal como lo establece la norma NTC 2680 (ICONTEC, 2013), determinando el $\mathrm{P}_{\text {valor }}$ previa definición de $p=0,5$ y q = 0,5, con un nivel de confianza de 95\%; según la citada norma, se considera ajustado para los 18 ensayos realizados, cuando se superan 14 aciertos. Para comparar las respuestas del panel en cada sesión de entrenamiento con los resultados emitidos por el panel de expertos en la prueba específica de análisis sensorial de café 
según metodología SCAA, se utilizaron pruebas de " $t$ " de Student para comparación de muestras independientes con nivel de confianza del 95\%. Finalmente, se procesaron de manera conjunta, todos los juicios emitidos tanto por panelistas en entrenamiento como por jueces expertos, mediante pruebas de análisis clúster para definir diferencias y similitudes de acuerdo a la bondad de agrupación. Todo el tratamiento estadístico de los datos se efectuó con el software Statgraphics 5.1 Plus (Magnugistics Inc., Rockville MD. USA)

\section{RESULTADOS Y DISCUSIÓN}

Los resultados de las pruebas de reconocimiento de aromas básicos en café están presentados en la Figura 1, como puede observarse, se presentó un incremento gradual progresivo en el reconocimiento de aromas por parte del grupo, a medida que avanzó el entrenamiento. En su conjunto, el grupo reconoció en promedio el 30,25\% de los aromas evaluados durante la primera sesión, llegando a reconocer de manera acertada el 69,75\% al finalizar la séptima sesión. Estos resultados pos- teriormente, facilitaran la calificación de los atributos fragancia y aroma de acuerdo con la metodología SCAA. Vale la pena resaltar la magnitud de la variabilidad de las apreciaciones dentro del grupo, indican heterogeneidad debida a que cada persona presenta diferente sensibilidad para los olores, al igual que los umbrales de olor de cada sustancia, los cuales son diferentes. La intensidad de los olores puede ser leve, débil o fuerte (Puerta, 2009).

En general, en paneles inexpertos se encuentra alta variabilidad de los juicios individuales con respecto al promedio del grupo. Situaciones similares han sido reportadas por Rossi (2001) y por Latreille et al.(2006) quienes establecieron la reproducibilidad y la fiabilidad como propiedades necesarias en paneles entrenados. Adicionalmente, y considerando la variabilidad natural en los juicios individuales, la metodología SCAA incluye al final de las rondas individuales, la denominada ronda de consenso en la que los jueces expresan abiertamente sus dictámenes y dirimen diferencias de opinión.

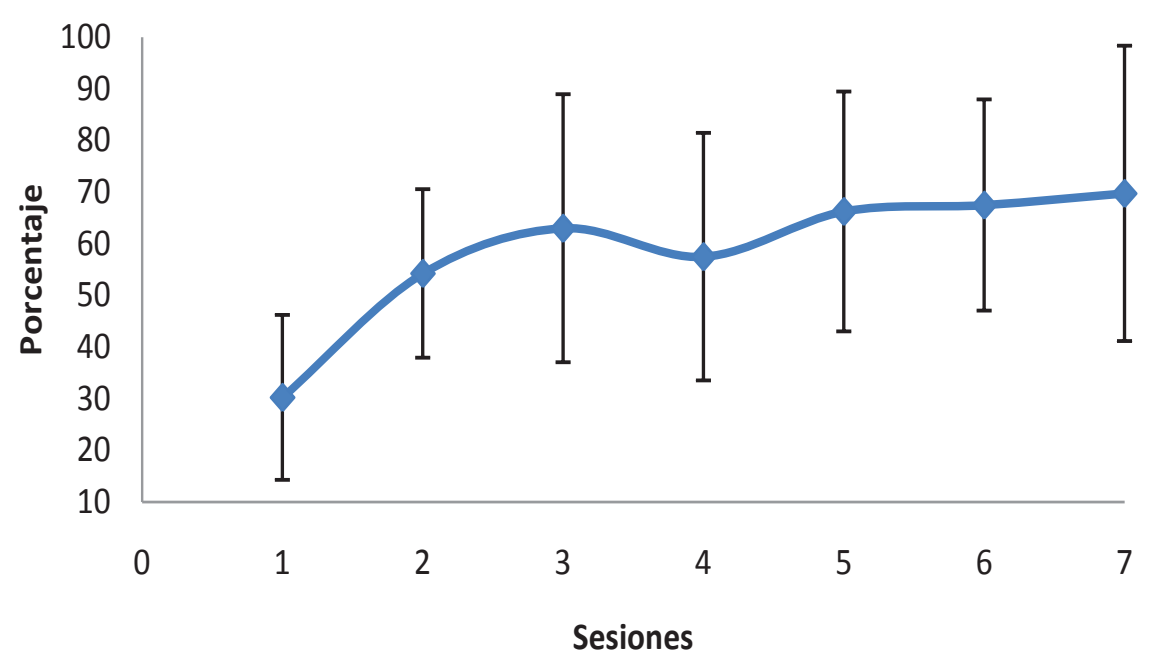

Figura 1. Resultados prueba de aromas básicos en café 
Con respecto a las pruebas de comparación pareada para reconocimiento de aromas básicos en café, en la Tabla 5, se presentan los resultados para las 18 pruebas aplicadas a cada panelista. Como puede verse, todos los panelistas obtuvieron 14 o más aciertos, lo que implica el cumplimiento en términos de la norma NTC 2680. De la misma forma, los $\mathrm{P}_{\text {valor }}$ obtenidos, indican que no existen diferencias estadísticamente significativas $\left(\mathrm{P}_{\text {valor }}>0,05\right)$ entre lo planteado en la prueba y lo expresado por el evaluador. Teniendo en cuenta que al momento de la aplicación de esta prueba, el equipo de panelistas ya habían superado las pruebas de instrucción básica en aromas y obtenido un importante nivel de avance en el reconocimiento de aromas con la nariz del café (Figura 1). Los resultados obtenidos (Tabla 5) sugieren que el panel ya está en condiciones de realizar análisis descriptivo en aromas de café.

Los resultados correspondientes a la prueba de umbral de detección de sabores realizada por el panel en la Tabla 6. Se destaca que cinco panelistas detectaron el sabor salado a concentraciones de $10^{-1}$, mientras que en menor proporción los panelistas detectaron concentraciones menores y algunos no lo percibieron. Los demás sabores evaluados fueron detectados solamente por la mitad de los panelistas a diferentes concentraciones para cada caso. Esta situación pudo presentarse por que se utilizaron concentraciones muy bajas y el grupo tuvo alguna dificultad asociada a trastornos del gusto pues pueden sentir sabores que no existen, no diferenciar los sabores o no percibir ningún sabor (Medlineplus, 2014a).

Tabla 5. Resultados prueba de comprobación en

reconocimiento de aromas básicos del café.

\begin{tabular}{cccccc}
\hline $\begin{array}{c}\text { Código } \\
\text { panelistas }\end{array}$ & $\begin{array}{c}\text { No. } \\
\text { ensayos }\end{array}$ & $\begin{array}{c}\text { No. } \\
\text { Aciertos }\end{array}$ & $\begin{array}{c}\text { No. } \\
\text { Fracasos. }\end{array}$ & $\begin{array}{c}\text { Probabilidad } \\
\text { obtenida }\end{array}$ & $\mathbf{P}_{\text {valor }}$ \\
\hline CC1 & 18 & 17 & 1 & 0,00007248 & 0,999927521 \\
CJ2 & 18 & 15 & 3 & 0,00376892 & 0,996231079 \\
CE3 & 18 & 15 & 3 & 0,00376892 & 0,996231079 \\
OE4 & 18 & 15 & 3 & 0,00376892 & 0,996231079 \\
OL5 & 18 & 16 & 2 & 0,00065613 & 0,999343872 \\
OD6 & 18 & 17 & 1 & 0,00007248 & 0,999927521 \\
SR7 & 18 & 14 & 4 & 0,01544189 & 0,984558105 \\
SL8 & 18 & 15 & 3 & 0,00376892 & 0,996231079 \\
TE9 & 18 & 14 & 4 & 0,01544189 & 0,984558105 \\
TD10 & 18 & 17 & 1 & 0,00007248 & 0,999927521 \\
\hline
\end{tabular}

Tabla 6. Resumen de prueba de umbral de detección.

\begin{tabular}{|c|c|c|c|c|c|c|c|c|c|c|}
\hline Concentración & $10^{-1}$ & $10^{-2}$ & $10^{-3}$ & $10^{-4}$ & $10^{-5}$ & $10^{-6}$ & $10^{-7}$ & $10^{-8}$ & $10^{-9}$ & $10^{-10}$ \\
\hline Sabores & \multicolumn{10}{|c|}{ Número de panelistas que detectaron un estímulo para cada dilución } \\
\hline Acido & - & - & 1 & 1 & - & - & 1 & 2 & - & - \\
\hline Amargo & - & 1 & - & 2 & - & 1 & - & 1 & - & - \\
\hline Salado & 5 & - & - & - & - & 1 & - & 1 & - & - \\
\hline Dulce & 2 & 1 & 1 & - & 1 & - & - & - & - & - \\
\hline
\end{tabular}


En la Figura 2 se reportan los resultados de la prueba de reconocimiento de los sabores básicos; teniendo en cuenta que a esta prueba se presentaron nueve panelistas. Los sabores salado, ácido y neutro fueron identificados por todos los asistentes, el sabor dulce fue identificado por ocho de los nueve panelistas y el sabor amargo fue identificado por cuatro de los nueve panelistas. Estos resultados están de acuerdo con lo reportado por Mantilla (2007), quien afirma que generalmente en este tipo de pruebas, el sabor amargo se confunde con el sabor ácido, dificultando su identificación. La dificultad para la identificación del sabor amargo, puede estar asociada a factores de salud, genéticos y fisiológicos. Por otro lado, la identificación de los sabores básicos es importante, pues éstos contribuyen en el sabor típico del café en la siguiente proporción: 40\% amargo, 35\% ácido, 15\% dulce y 10\% salado (Puerta, 2009).

Los resultados de la aplicación de las tres sesiones de la metodología SCAA para definir los perfiles descriptivos de las muestras de café, se presentan en la Tabla 7. Durante la primera sesión se evaluaron siete muestras de café, durante la segunda seis muestras y en la tercera sesión ocho muestras. Se pueden observar los $\mathrm{P}_{\text {valor }}$ resultantes de la comparación mediante la prueba de Student ( $\mathrm{t}$ ), de las puntuaciones entregadas por el panel en entrenamiento con los juicios emitidos por el panel de expertos, para cada uno de los 10 atributos de calidad evaluados.
En la primera sesión, se encontró que en seis de los 10 atributos evaluados no se presentaron diferencias estadísticamente significativas $\left(\mathrm{P}_{\text {Valor }}>\right.$ $0,05)$ entre el juez experto y los panelistas, mientras que en los atributos "fragancia/aroma", "uniformidad", "balance" y "taza limpia", se encontraron diferencias estadísticamente significativas $\left(\mathrm{P}_{\text {Valor }}<0,05\right)$. Es importante destacar que al finalizar cada catación, se realizó una discusión de los resultados con los panelistas sobre los errores comunes al iniciar este tipo de entrenamiento. En la segunda sesión, en todos los atributos evaluados en la prueba de comparación se obtuvieron $\mathrm{P}_{\text {valor }}$ mayores que 0,05 , lo que indica que no existen diferencias estadísticamente significativas entre los panelistas y los jueces expertos. Este resultado es satisfactorio ya que los panelistas se han acercado a las calificaciones hechas por el juez en cada una de las muestras. Finalmente, después de la tercera sesión de entrenamiento, todos los $\mathrm{P}_{\text {valor }}$ resultaron en magnitudes mucho mayores que 0,05 , lo que indica el ajuste entre las valoraciones de los jueces expertos y los panelistas; los $\mathrm{P}_{\text {valor }}$ para los atributos uniformidad, balance, taza limpia y dulzor, correspondientes a la tercera sesión, fueron asumidos como uno porque no presentaron variabilidad entre la calificación del juez experto y los panelistas, es decir todos coincidieron en la valoración, indicando ajuste perfecto sin variabilidad.

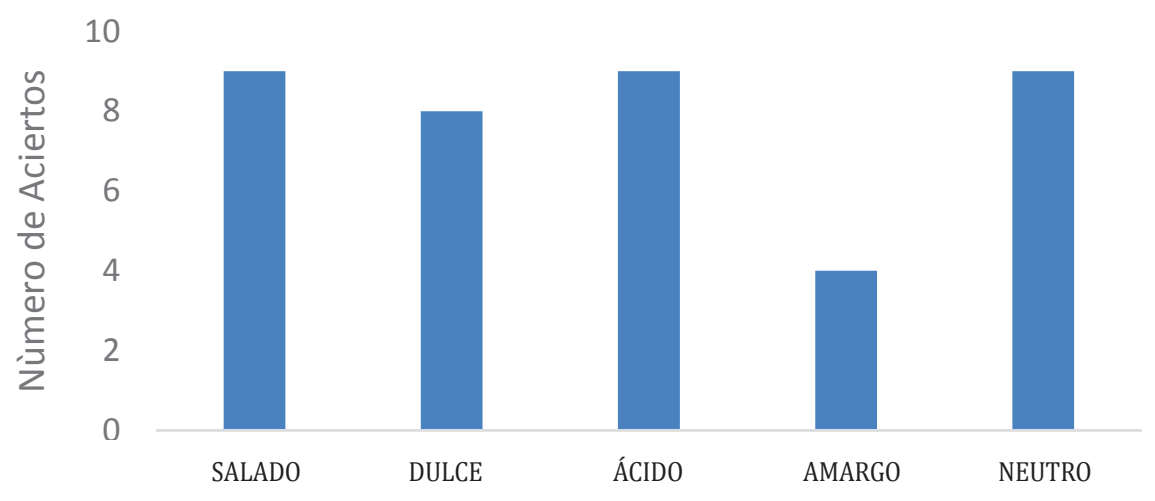

Figura 2. Identificación de los sabores básicos en café 
Tabla 7. Prueba de Student ( $t$ ) para la comparación entre panel en entrenamiento y jueces expertos.

\begin{tabular}{lccc}
\hline \multirow{2}{*}{\multicolumn{1}{c}{ Atributo }} & \multicolumn{3}{c}{$\mathbf{P}_{\text {valor }}$ para cada sesión } \\
\cline { 2 - 4 } & $\mathbf{1}$ & $\mathbf{2}$ & $\mathbf{3}$ \\
\hline Fragancia/Aroma & 0,003 & 0,589 & 0,648 \\
Sabor & 0,064 & 0,463 & 0,159 \\
Sabor residual & 0,17 & 0,792 & 0,669 \\
Acidez & 0,14 & 0,597 & 0,541 \\
Cuerpo & 0,078 & 0,289 & 0,808 \\
Uniformidad & 0,029 & 0,851 & 1 \\
Balance & 0,045 & 0,851 & 1 \\
Taza limpia & 0,029 & 0,0518 & 1 \\
Dulzor & 0,051 & 0,0518 & 1 \\
Puntaje Global & 0,644 & 0,363 & 1 \\
\hline Totales & 0,016 & 0,577 & 0,722 \\
\hline
\end{tabular}

Pvalor para comparación de medias. Prueba t Student. Nc=95\%

El análisis multivariado de clasificación (Método Clúster) se presenta en las Figuras 3 y 4 . Este tipo de análisis permitió determinar el desempeño a través del análisis de interacción entre el producto y el panelista (Acosta, 2010). La Figura 3 muestra las diferencias que existen entre las calificaciones hechas por los panelistas y los jueces expertos durante la primera sesión de entrenamiento; en este caso, se generaron dos conglomerados a partir de las valoraciones emitidas. En la Figura 4 que corresponde a la tercera sesión se generó un solo clúster indicando que tanto los jueces entrenados como los panelistas confluyen en sus juicios, lo cual es una muestra de la efectividad del protocolo utilizado para el entrenamiento.

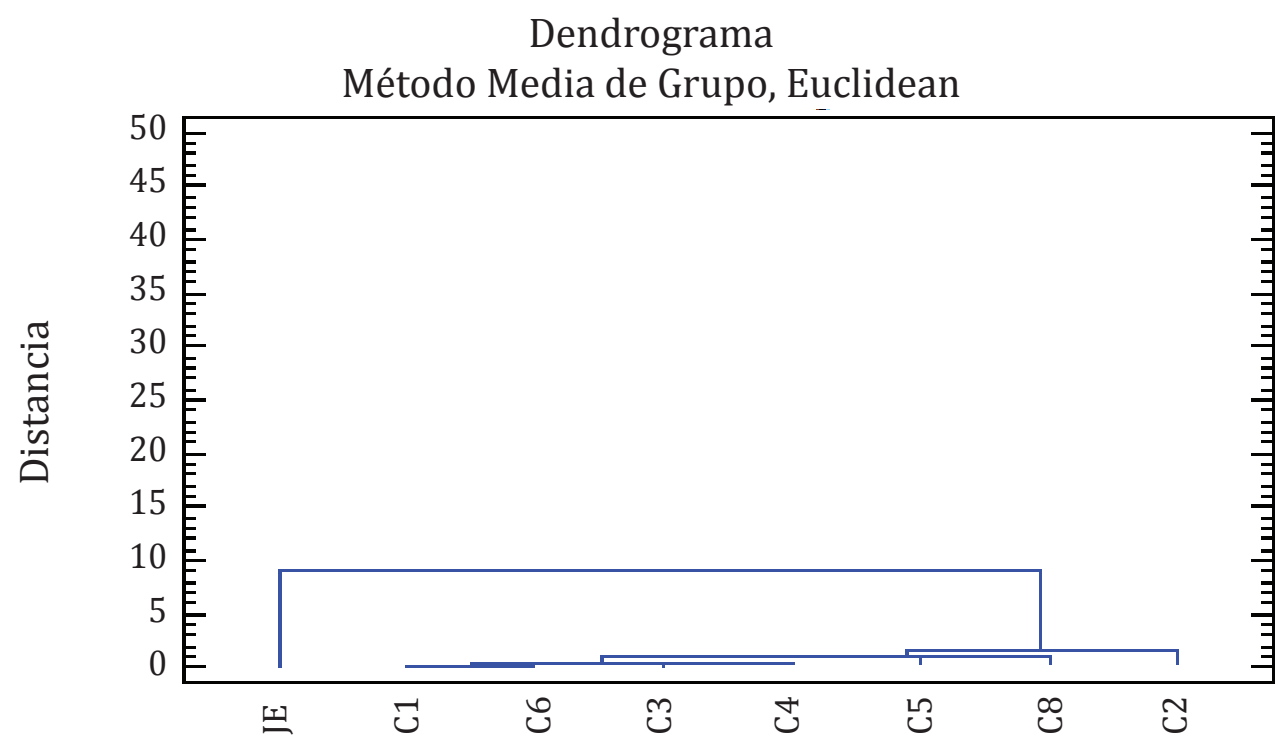

Figura 3. Diagrama de dispersión de conglomerados para una de las muestras de la primera sesión. 


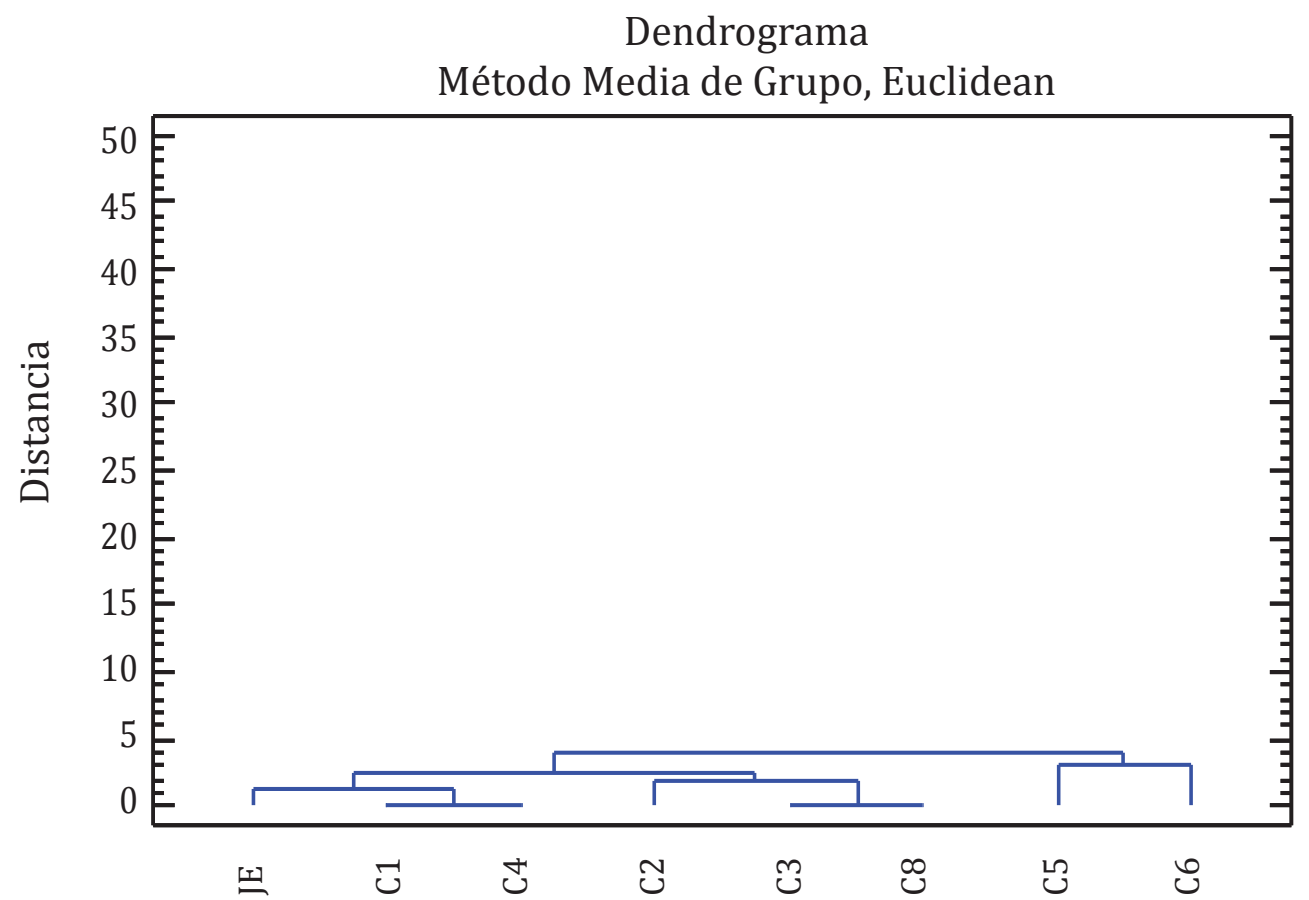

Figura 4. Diagrama de dispersión de conglomerados para una de las muestras de la tercera sesión.

\section{CONCLUSIONES}

La secuencia en las etapas definida para el entrenamiento de panelistas sin experiencia en evaluación sensorial de café resultó ser adecuada, en solo tres sesiones de entrenamiento específico se superaron las diferencias entre los juicios emitidos por el panel en proceso de entrenamiento $\mathrm{y}$ los jueces expertos.

El análisis de conglomerados confirma la efectividad del método utilizado; la conformación de un solo clúster al final del entrenamiento indica mínima variabilidad entre los panelistas y un juez experto.

El tiempo total del entrenamiento fue de 72 horas en un periodo de tres meses, durante este periodo se realizaron los procesos de reclutamiento, motivación, adiestramiento teórico y entrenamientos básico y específico y comprobación del adiestramiento.
En el análisis sensorial de cafés especiales, la inclusión del reconocimiento de defectos que pueden llegar a descalificar una muestra, resulto muy importante dentro del entrenamiento; principalmente en lo relacionado con el reconocimiento de los defectos de "reposo" y "fermento".

\section{REFERENCIAS BIBLIOGRÁFICAS}

ACOSTA, M. C. 2010. Análisis multivariado para la identificación de componentes Generadores de sabor y aroma en productos alimenticios. Disponible en: http://www.bdigital.unal.edu.co/2536/1/carolinaacostamunoz.2010.pdf, consulta: junio, 2014.

BOHÓRQUEZ, D., AGUDELO, J. I., GÓMEZ, J. F., CUELLAR, L. A., MARÍN, O. A., PACHÓN, R. y MENDOZA G. A. 2005.Identificación de algunos compuestos responsables del sabor en el café. Revista colombiana de física. 37(1):13 - 16.

CALLEJO M.J., VARGAS-KOSTIUR M.E. y RODRÍGUEZ-QUIJANO, M. 2015. Selection, training and validation process 
of a sensory panel for bread analysis: Influence of cultivar on the quality of breads made from common wheat and spelt wheat. Journal of cereal science. 61:55 - 62 .

CASTRO, J. B., RAMANATHAN, A. y CHENNUBHOTLA, C. S. 2013. Categorical Dimensions of Human Odor Descriptor Space Revealed by Non-Negative Matrix Factorization. PLOS Open Access Journal. 8 (9): e 73289. Disponible en: http://www.ncbi.nlm.nih.gov/pmc/ articles/PMC3776812/; consulta: julio, 2014.

DORRI, Y., SABEGHI, M. y KURIEN, B. T. 2007. Awaken olfactory receptors of humans and experimental animals by coffee odorants to induce appetite. Med. Hypotheses, 69(3): 508 - 9.

DUARTE, C., ORTEGA, A. y TRUJILLO, L. 2008. Metodología para la formación de comisiones de evaluación sensorial en café. Instituto de Investigaciones para la Industria Alimentaria. La Habana Cuba. 7 p.

FERIA, A. M. 2002. Examining the case of green coffee to illustrate the limitations of grading systems/expert tasters in sensory evaluation for quality control. Disponible en: http://www.sciencedirect.com/ science/article/pii/S0950329302000289\#; consulta: julio, 2014.

GONZÁLEZ, C. J., MIGUELEZ, DE LA MONTAÑA, J. y MIGUEZ B. M. 2002. Estudio de la percepción de sabores dulce y salado en diferentes grupos de la población. Universidad de Vigo. España. 3 p.

HENAO, J.D. 2009. Mucilagem residual em café cereja descascado e seus efeitos na qualidade da bebida e na produção de cafés especiais. Tesis Doctoral. Universidade Estadual De Campinas: Campinas, Brasil. 354 p.

INSTITUTO COLOMBIANO DE NORMAS TÉCNICAS (ICONTEC). 2013. Norma Técnica Colombiana (NTC 2680). Análisis sensorial. Metodología prueba de comparación pareada. 2 - 4 p.

INSTITUTO COLOMBIANO DE NORMAS TECNICAS (ICONTEC). 1998. Norma Técnica Colombiana (NTC 4503). Análisis Sensorial. Metodología iniciación y entrenamiento de evaluadores y reconocimiento de olores $12 \mathrm{p}$.
KREUTZMANN, S., THYBO, A. y BREIDE, W. 2007. Training of a sensory panel and profiling of winter hardy and coloured carrot genotypes. Food Quality and preferences. 13(3):482 - 489.

LATREILLE, J., MAUGER, E., AMBROISINE, L., TENENHAUS, M., VICENT, M., NAVARRO, S. y GUINOT, C. 2006. Measurement of the reliability of sensory panel performances. Food quality and preferences. 17:369 375.

MAGALHÃES, M. 2002. Caracterização sensorial da bebida de café (coffea arabica l.): análise descritiva quantitativa, análise tempo-intensidade e testes afetivos. Tesis de grado. Universidade Federal de Viçosa: Minas Gerais - Brasil. 181 p.

MANTILLA S. M. J. 2007. Informe de prácticas profesionales. Disponible en: http://www.dspace.espol.edu.ec/ bitstream/123456789/9018/3/Informe\%20de\%20 Practicas\%20Profesionales\%20ALASA.pdf. Consulta: febrero, 2014.

MEDLINEPLUS. 2014a. Trastornos del gusto y del olfato. Disponible en: http://www.nlm.nih.gov/medlineplus/spanish/tasteandsmelldisorders.html; consulta: junio, 2014.

MONTENEGRO, G., GÓMEZ, M., PIZARRO, R. CASAUBON, G. y PEÑA, R. 2008. Implementación de un panel sensorial para mieles chilenas. Ciencia e Investigación Agraria. 35(1):51 - 58 .

PUERTA, G. I. 2009. Los catadores de café. Avances técnicos 381. Centro Nacional de Investigaciones de Café. CENICAFE. Chinchiná_Caldas. Colombia. 12p.

RENDON, M Y. FERREIRA, S. H. P., SCHOLZ, M. B. y BACCETI, M. A. 2007. Uso de Escala de qualidade e escala de intensidade para avaliação de bebidas de café. Disponible en: http://www.sbicafe.ufv.br/bitstream/handle/10820/1830/179995_Art241f.pdf?sequence=1; consulta: junio, 2014.

RODRÍGUEZ A., LEÓN R. y PADILLA, O. 1995. Vademécum del tostador colombiano. Sección B. evaluación sensorial. Quitan edición. Colombia. 263 p.

ROSI, F. 2001. Assessing sensory panelist performance using repeatability and reproducibility measures. Food quality and preferences. 12, 467 - 479. 